\title{
Use of artificial neural network for spatial rainfall analysis
}

\author{
Tsangaratos Paraskevas*, Rozos Dimitrios and Benardos Andreas \\ School of Mining and Metallurgical Engineering, National Technical University of Athens, Athens, Greece. \\ ${ }^{*}$ Corresponding author. e-mail: ptsag@metal.ntua.gr
}

In the present study, the precipitation data measured at 23 rain gauge stations over the Achaia County, Greece, were used to estimate the spatial distribution of the mean annual precipitation values over a specific catchment area. The objective of this work was achieved by programming an Artificial Neural Network (ANN) that uses the feed-forward back-propagation algorithm as an alternative interpolating technique. A Geographic Information System (GIS) was utilized to process the data derived by the ANN and to create a continuous surface that represented the spatial mean annual precipitation distribution. The ANN introduced an optimization procedure that was implemented during training, adjusting the hidden number of neurons and the convergence of the ANN in order to select the best network architecture. The performance of the ANN was evaluated using three standard statistical evaluation criteria applied to the study area and showed good performance. The outcomes were also compared with the results obtained from a previous study in the area of research which used a linear regression analysis for the estimation of the mean annual precipitation values giving more accurate results. The information and knowledge gained from the present study could improve the accuracy of analysis concerning hydrology and hydrogeological models, ground water studies, flood related applications and climate analysis studies.

\section{Introduction}

A key component in any hydrologic or hydrogeological analysis, planning and management of water resources is the accurate estimation of the water balance (Goncalves and Alpuim 2006; Wilk et al. 2006). This requires an accurate estimation of rainfall volumes and their spatial distribution. However, such data are recorded as observational data in locations over typically sparse networks and may show complex and nonlinear variation with latitude, longitude, altitude and probably other unknown variables. Specifically, precipitation distribution can be rather different from coast to inland, high altitude to low altitude, upper slope to down slope; however, it increases with elevation, a phenomenon referred to as orographic effect (Hutchinson 1968; Vuglinski 1972; Daly et al. 1994; Martínez-Cob 1995; Goovaerts 2000; Kyriakidis et al. 2001; Naoum and Tsanis 2004).

In order to make more accurate estimations from such sparse and partial data, rainfall data and their distribution can be estimated through spatial interpolation techniques. These interpolating techniques are applied to generate continuous surfaces, assuming that the distance or direction between sample points reflects a spatial correlation which is capable of expressing the variations in the surface. Unfortunately, potentially resolvable errors remain where the trend is nonlinear and show a complex and temporally varying relationship with the underlying landscape. Since accurate

Keywords. Artificial neural networks; precipitation data; spatial analysis; GIS. 
precipitation estimation is becoming an important issue for the scientific community, many different interpolation approaches, globally (e.g., partial thin plate splines, trend surface) and locally (e.g., inverse distance weighting, ordinary and universal kriging) and computational methods have been proposed in the past two decades (Jeffrey et al. 2001; Vicente-Serrano et al. 2003; Lloyd 2005; Chu et al. 2008; Hofstra et al. 2008; Kong and Tong 2008). Among them, estimating with Artificial Neural Network (ANN) has received increasing interest.

ANN are being proposed as an alternative method for spatial interpolation, as they exhibit good performance in recognizing patterns in datasets (Snell et al. 2000; Bryan and Adams 2002). The ANN approach makes no assumptions regarding the nature of the spatial data, such as stationarity and handle nonlinear relationships (Openshaw and Openshaw 1997). It is not needed to specify a particular variogram model as kriging method assumes (Rigol et al. 2001).

In particular, neural networks have been widely applied to model many nonlinear hydrologic processes, particularly rainfall estimation (Sivapragasam et al. 2001; Nourani et al. 2009; Kajornrit et al. 2012).

However, neural networks suffer from a number of shortcomings that have to do with the ability of generalizing and avoiding overfitting while training the network. The network learns specific random features of the training data that have no causal relation to the target function. It learns, in other words, details of the training dataset that do not increase knowledge and the ability to predict unknown features (Hagan et al. 1996). As a result, poor generalization, thus poor performance is recorded (Maren et al. 1990; Rojas 1996). In our case, the available data are characterized by the small number, the scattered spatial distribution are affected by noise, producing even more poor performance. Improvements in the ability of generalization and avoid overfitting can be achieved by determining the network architecture.

Essentially, the main objective of this study was to develop a trial-error procedure, in order to determine first, the number and connectivity of hidden layer nodes and second, the number of training epochs that ensures that overfitting does not occur. By this, the optimum network architecture was chosen, ensuring that the developed ANN will achieve better generalization and optimum performance.

The methodology was implemented in order to evaluate the spatial distribution of mean annual precipitation in Achaia County, Greece with adequate accuracy within the boundary of a specific catchment area.

\section{Materials and methods}

\subsection{The study area and the available data}

The County of Achaia is located at the NW part of Peloponnesus, covering an area of approximately $3274 \mathrm{~km}^{2}$ (figure 1). Despite its coastal extension, it can be considered as one of the most mountainous regions of Greece, since over $60 \%$ of its total area is highland with three main ranges (Panahaikon, Erymanthos and Helmos). The County of Achaia is characterized by high precipitation values; however, the prefecture faces a qualitative and quantitative water problem. In recent years there has been an increase in overexploitation of groundwater aquifers in the lowland regions and especially in the coastal zones. In addition, there are a significant number of agricultural and industrial activities in the county that demand great amounts of water supply (Rozos et al. 2008).

The spatial distribution of the available 23 observation stations throughout the Achaia County is considered satisfactory (figure 1). At least one station over $600-850 \mathrm{~km}^{2}$ for plain areas and at least one station over $100-250 \mathrm{~km}^{2}$ for mountainous areas could be found. The available data are monthly precipitation values that cover different periods of the hydrological year. However, there is a significant common period, approximately 25 years, from which the mean annual precipitation values of each station were obtained (Rozos et al. 2008).

A specific area, Selinounda catchment, has been considered for evaluating the performance of the developed ANN. The surface extent of the catchment is approximately $377 \mathrm{~km}^{2}$, having a mean altitude of $795 \mathrm{~m}$ (figure 2). The terrain parameters were obtained from a Digital Elevation Model $(\mathrm{DEM})$ with $25 \times 25 \mathrm{~m}$ grid cell. The ANN used as input variables the latitude and longitude values (Coordinate System, EGSA' 87) of each observation station, their elevation values in meters, and has a target variable, the mean annual precipitation values in $\mathrm{mm}$ that each station has. In the specific catchment five observation points were located.

\subsection{Artificial neural network}

Artificial neural network are information processing systems that have the ability to discover knowledge automatically using the mode of learning analogous to human brain and neural biology. They are a 'computational mechanism able to acquire, represent and compute a mapping, from one multivariate space of information to another, given a set of data representing that mapping' (Garrett 1994). They are typically composed of layers of neurons or processing elements, which are interconnected 


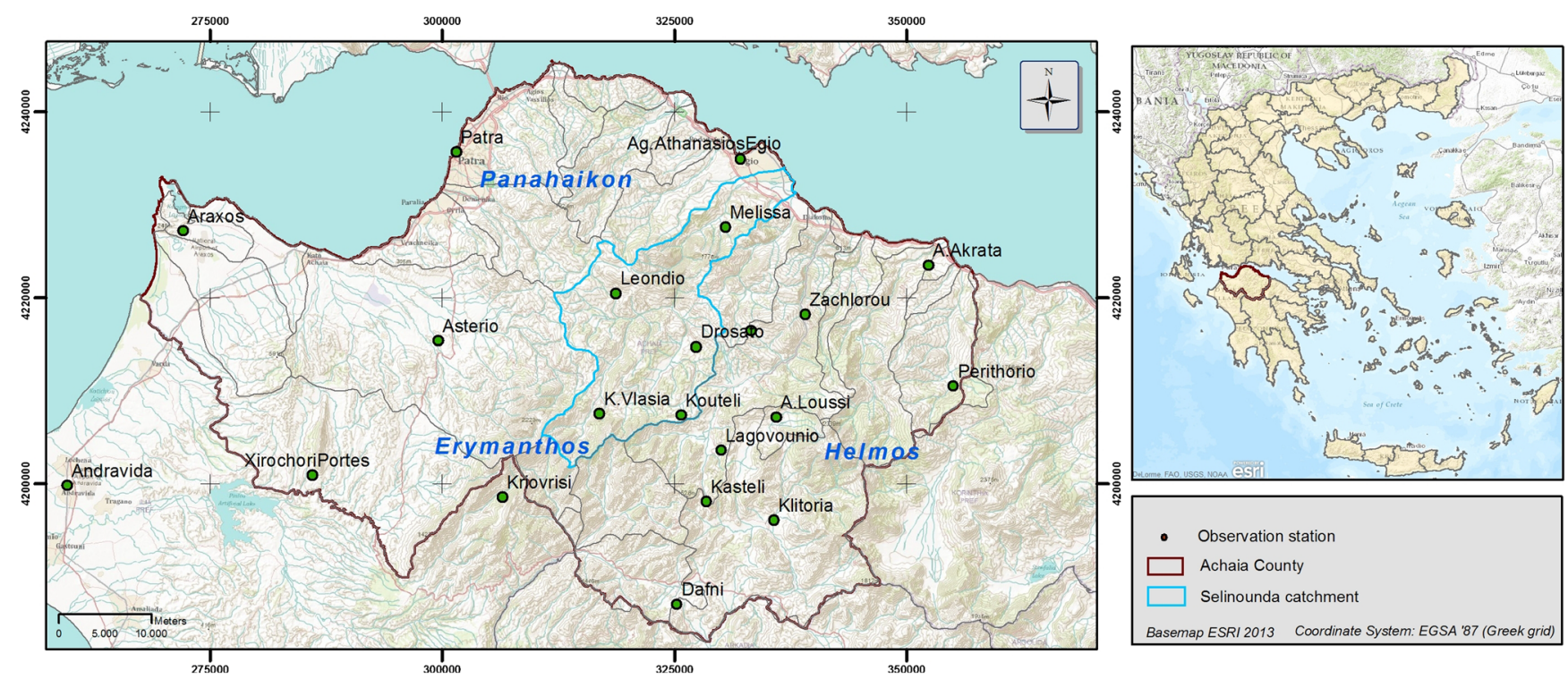

Figure 1. The study area.

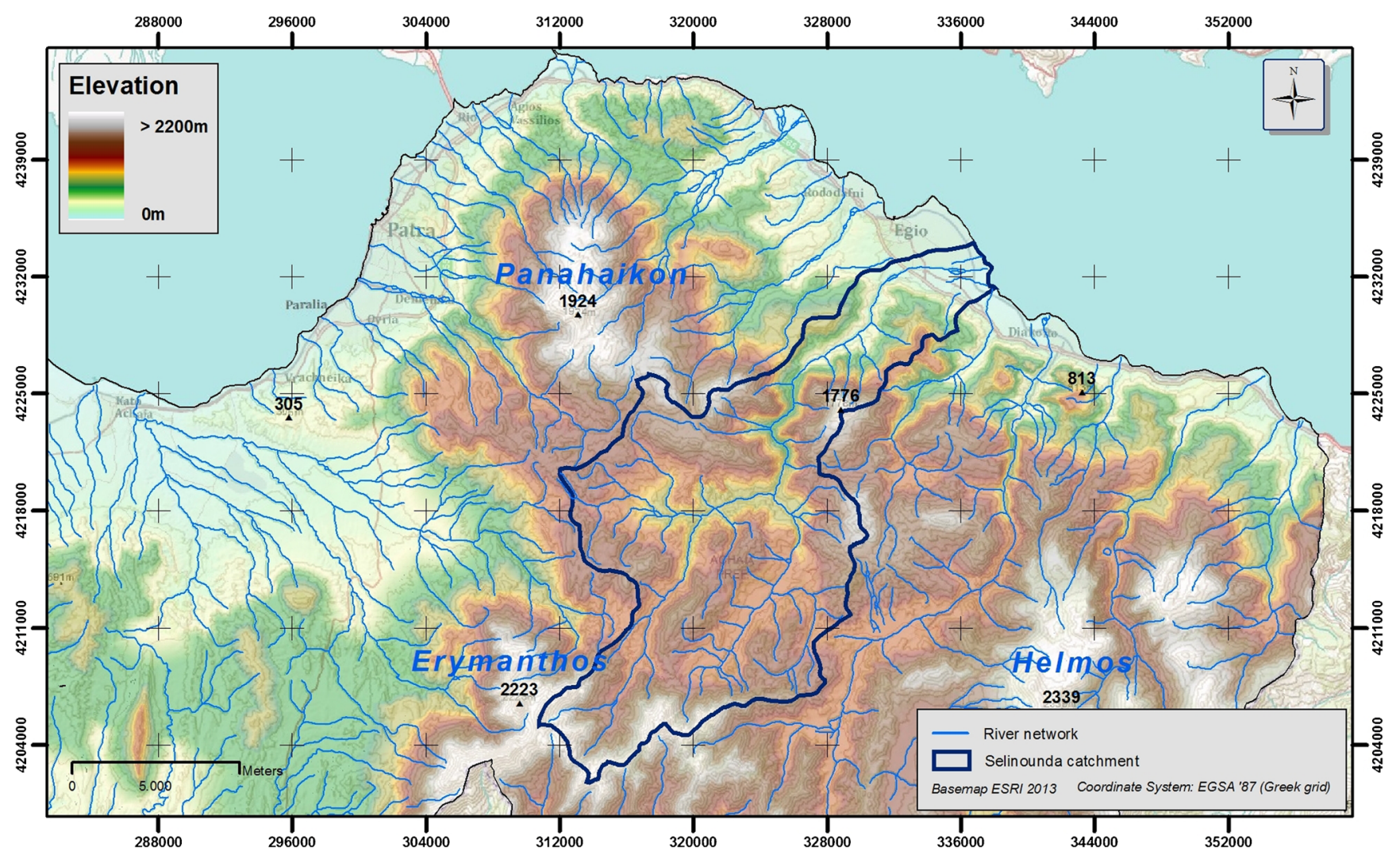

Figure 2. The Selinounda catchment.

by set of correlation weights. They are recognized as a complex nonlinear mathematical function that converts input data to a desired output.

In Multilayer Perceptron Neural Network, such as the one that is presented in this study, there is always an input layer, a hidden layer and an output layer as shown in figure 3 (Rumelhart et al.
1986). The first layer of the network or input layer contains a node for each of the input variables. The input variables are analogous to the independent variables in multiple regression techniques. The second layer, the hidden layer, consists of nodes that allow complexities to develop among input nodes. The last layer of the network or 


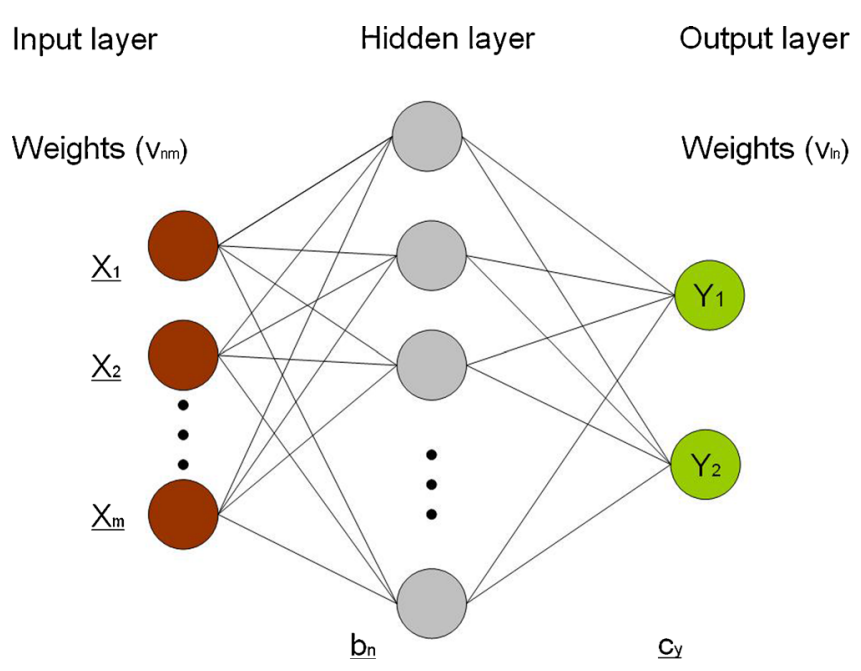

Figure 3. The feed-forward multilayer artificial neural network.

output layer contains $t$ nodes, one for each output type.

The back-propagation algorithm is one of the most commonly used algorithms that follow the multiple-layer architecture with fully connected interactions between layers. Typically, one or more hidden layers are included to enable the network to learn complex tasks and training is based on an error-correction learning rule, a general rule of the least-mean-square (LMS) algorithm (Rumelhart et al. 1986).

During the training phase, the hidden and output layer neurons process their inputs by multiplying each input by a corresponding weight, summing the product, and then processing the sum using a nonlinear transfer function to produce a result. An ANN learns by adjusting the weights between the neurons in response to the errors between the actual output values and the target output values. At the end of this training phase, the neural network provides a scheme that should be able to predict a target value from a given input value. In general, the method used to estimate the values of the synaptic weights, trains the multilayered network until some targeted minimal error is achieved between the desired and actual output values. Once the training is complete, the classification phase follows, where the network is used as a feed-forward structure to produce a classification for the entire data.

\subsection{ANN development - methodology}

The developed methodology involves the following phases:

- phase of data processing,

- phase of determining the architecture of the network,
- predictive phase,

- spatial analysis phase (figure 4).

In the phase of data processing, a simple text file which contained the ID number, topographic position and mean annual precipitation values of each observation station was imported into the system in order to normalize the data and produce the necessary training, and validation datasets.

During the training phase, all the necessary processing elements, number of neurons, number of hidden layers, learning and momentum rate, are all set with user defined initial values. The algorithm enables an optimized function that monitors in parallel the validation data during the training phase and terminates the process of learning when a certain threshold is achieved. It also implies a method for handling the available training dataset, producing a series of different outcomes from schemes with different processing elements. The results are presented through a matrix, with the help of which we choose the best predictive scheme. The parameters of the ANN are saved while passing to the testing phase.

An untrained set of data that is also imported as a simple text file, evaluates the performance of the ANN. The results of the ANN are shown through the table that contained statistical performance evaluation criteria.

In the next phase, the predictive phase, the thematic GIS layer that has information concerning topographical aspects, is introduced to the ANN in the specific form that is required. After running the algorithm, the outcomes are converted again into a grid file.

The final phase, the spatial analysis, is the phase where the grid file is imported into a GIS package and the generated continuous surface is inspected visually for inconsistencies or errors while statistical parameters are derived.

\subsection{Data processing}

During this phase the datasets were normalized so that all variables received equal attention during the learning process. The normalized values ranged between 0.1 and 0.9 . Also in this phase, the entire database was randomly separated into two groups: one, donated as Training Dataset (TrD), for training the model and a second one, donated as Validation Dataset ( $\mathrm{VaD})$. In the $\mathrm{VaD}$, we needed to include at least one station that actually appeared within the boundaries of the research area. The process of random separation ended only after at least one station appeared into the $\mathrm{VaD}$. The size of each dataset was about $80 \%$ for $\operatorname{TrD}$ and $20 \%$ for $\mathrm{VaD}$ (Shanin et al. 2004). 


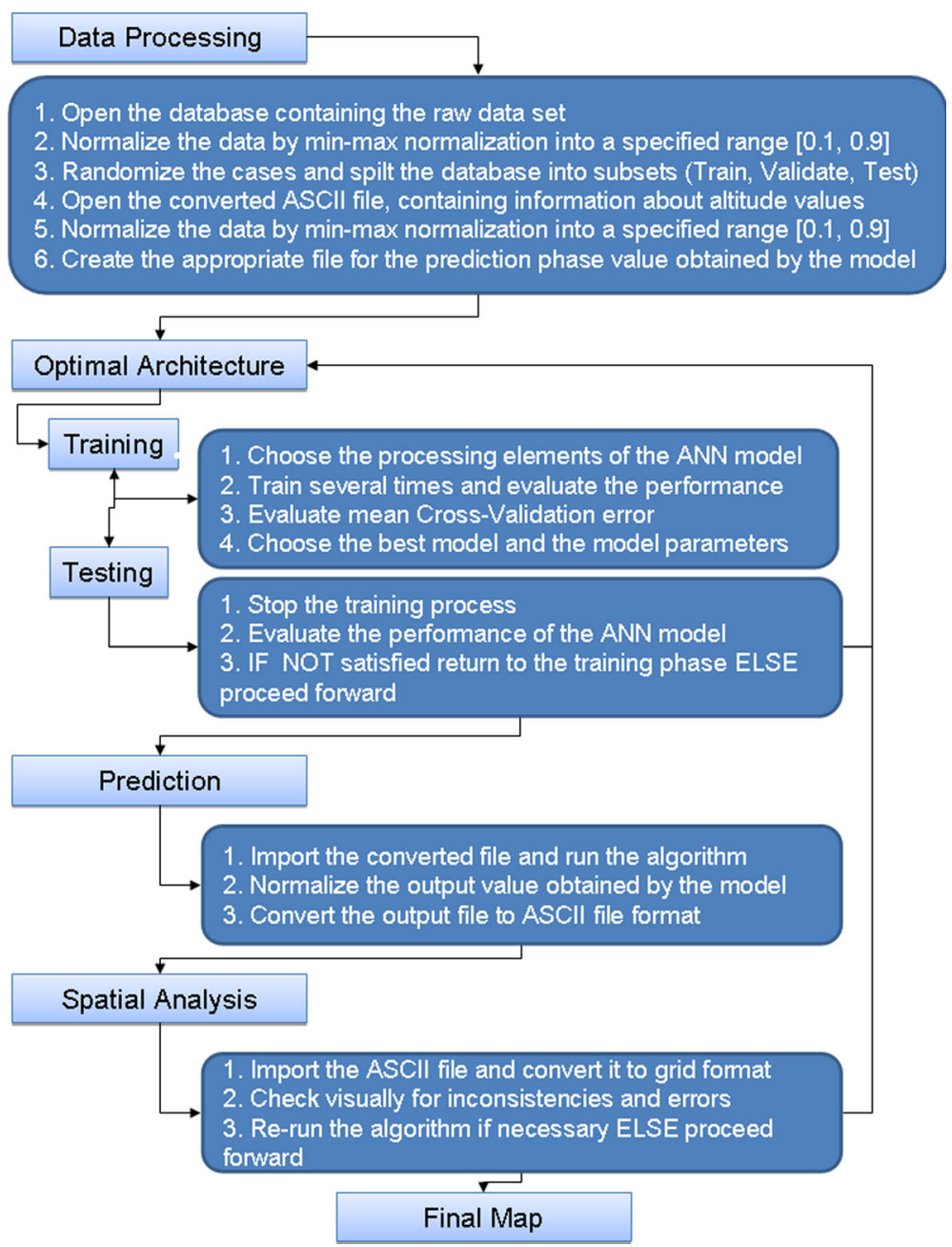

Figure 4. Schematic presentation of the developed framework.

\subsection{Determining the best architecture}

During the development of the network, it is required to select the optimum number of layers and the number of hidden nodes. However, many researchers have stated that there is no direct and precise way of determining the optimum number of nodes in each hidden layer. If the number is too small the network is not able to solve the learning task, while if the number is too large the problem of overfitting occurs (Benardos and Kaliampakos 2004).

Overfitting may also occur during the training phase. As the training epochs increase, the performance of the network on the training examples increases too. However, the performance on unseen data may become worse.

The developed methodology begins by applying an initial number of hidden neurons, equal to the number of the input variables, to the hidden layer. It ends when each of the processing elements reaches the upper boundaries of the network parameters and actually no significant improvement in the ANN's performance is achieved. By this the algorithm produces a series of different outcomes from networks with different processing elements. The results are presented though a matrix, with the help of which we choose the best predictive network.

The developed methodology uses a stopping criterion to decide when to terminate the training phase, a stopping threshold, applied on the TrD. To ensure that during the training and testing phase, we applied the Leave One Out Cross-Validation (LOOCV) technique, on the $\operatorname{TrD}$, creating by these another dataset, the Testing Dataset. LOOCV is a $k$-fold cross-validation technique, where $k$ equals the size of the dataset (Amari et al. 1997). It assumes a single observation from the original sample as the testing data, while the remaining observations are considered as the training data. It is mainly used in settings where the objective is to gain a prediction, and estimating how accurately a predictive model will perform in practice 
(Kohavi 1995). Cross-validation has been widely applied in studying the accuracy of prediction methods in precipitation fields.

This process is repeated such that each observation in the $\operatorname{TrD}$ will be used once as testing data. It gives to the network the option of gaining the most from the $\operatorname{TrD}$, since the number of the spatially scattered observation stations is limited. The form of the technique is as follows:

For $k=1$ to $r$ (where $r$ is the number of training points)

- Temporarily remove the $k$ th data point from the training set.

- Train the learning algorithm on the remaining $r-1$ points $(\mathrm{TrD})$.

- Test the removed data point and note your error ( $\mathrm{TeD}$ ).

- Calculate the mean error over all $r$ data points.

The TrD set was used to train the ANN, whereas the $\mathrm{TeD}$ was used to evaluate the performance of the model at the various stages of the training phase. The $\mathrm{VaD}$ set is used to evaluate the performance of the trained model in the deployed environment.

The learning process terminates when the mean absolute percentage error (MAPE) calculated from the $\operatorname{TrD}$ is reduced to a minimum or acceptable level. The MAPE is a measure of accuracy in statistics, specifically trending. It usually expresses accuracy as a percentage, and is defined according to the formula:

$$
\mathrm{MAPE}=100 \% \frac{1}{n} \sum_{t=1}^{n}\left|\frac{A_{t}-F_{t}}{A_{t}}\right|
$$

where $A_{t}$ is the actual value and $F_{t}$ is the forecast value.

When the process reaches the predefined threshold, the MAPE for the validation dataset is calculated. The average value of the MAPE for the $\operatorname{TrD}$ and the MAPE for the TeD value is calculated and the processing elements of the network are stored. The same procedure is performed for a different number of hidden neurons and stop training thresholds. The network that has the lowest average value is selected as being more accurate and with a better ability of generalizing.

\subsection{Validating the model}

By applying the proposed procedure, we were able to adjust the acceptable MAPE-TrD in a range of $5-10 \%$ and perform the LOOCV technique in order to evaluate the network with the lowest average value. The performances of the different configurations are evaluated by statistical indices, the error bias, the root mean square error (rmse) and the correlation index (corr).

\section{Results and discussion}

The learning rate of the ANN was set to 0.88 and the monument rate was set to 0.8 while the initial weights were randomly selected between the space $[-0.5,0.5]$. As already mentioned, the main objective is to enhance the network's learning ability to generalize and predict more accurately from unknown points. In general, LOOCV is very expensive from a computational point of view because of the more number of times the training process is repeated. However, since the database of the observation station had less number of stations, the above technique was applied in order to choose the best predictive ANN network. The process begins by applying an initial number of hidden neurons, with one hidden layer and a certain minimal early stopping training threshold and ends when each of the processing elements reaches the upper boundaries of the model parameters. This process was repeated 10 times and the mean values of each statistical parameter are obtained. Analyzing the results during training, it can be observed that the best performed model is the one with 10 hidden neurons with stopping threshold of $6 \%$. The average MAPE was found minimal when the certain topology was chosen (table 1 ).

Passing to the predictive phase, the ASCII file containing topographic position information is imported to the system. Running the algorithm, the unknown values were calculated and by the

Table 1. Results of the average mean absolute percentage error value.

\begin{tabular}{|c|c|c|c|c|c|c|}
\hline \multirow[b]{2}{*}{ Network } & \multicolumn{6}{|c|}{ Stopping threshold } \\
\hline & $5 \%$ & $6 \%$ & $7 \%$ & $8 \%$ & $9 \%$ & $10 \%$ \\
\hline $3-6-1$ & 9.5676 & 9.5790 & 10.0975 & 9.9869 & 10.4790 & 10.6657 \\
\hline $3-7-1$ & 10.1401 & 10.1114 & 9.7432 & 10.0523 & 10.4475 & 10.7182 \\
\hline $3-8-1$ & 9.9010 & 10.1712 & 10.2287 & 10.2634 & 10.1659 & 10.4977 \\
\hline $3-9-1$ & 10.2844 & 10.5838 & 10.1742 & 9.6809 & 10.0780 & 10.6372 \\
\hline $3-10-1$ & 10.5659 & 9.5407 & 10.3863 & 10.2110 & 10.0127 & 10.5203 \\
\hline $3-11-1$ & 9.9879 & 10.5979 & 10.5208 & 9.8986 & 9.8384 & 10.7653 \\
\hline
\end{tabular}


end of the process the outcomes were converted into grid format. In the final phase, specific spatial parameters were calculated while the generated surface represents the mean annual precipitation throughout the Selinounda catchment area (figure $5 \mathrm{a}$ and $\mathrm{b}$ ). The final thematic precipitation layer has been compared with the one calculated during a previous study (Rozos et al. 2008). In that study a linear regression analysis was employed to interpolate the mean annual precipitation that was expressed through the following formula:
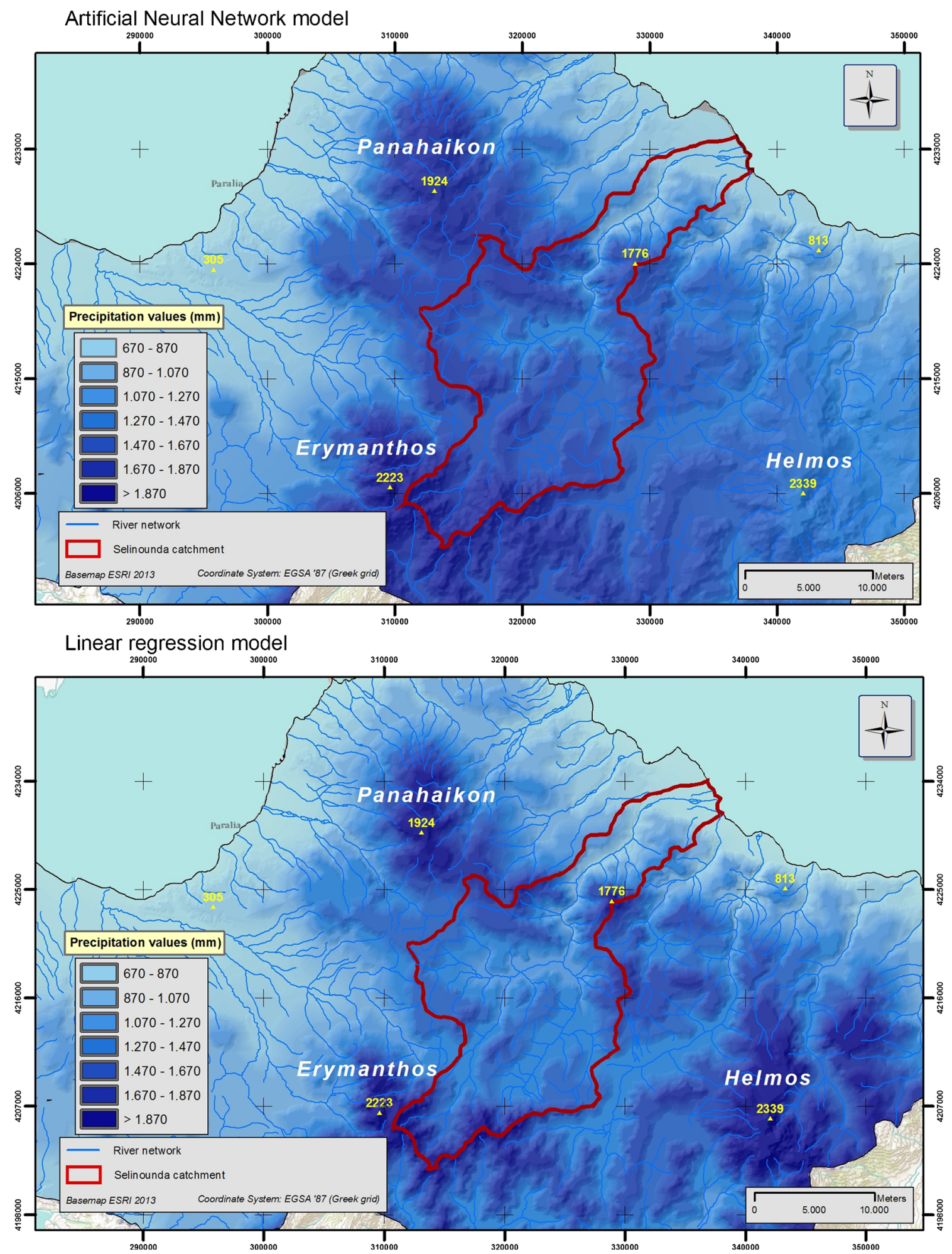

Figure 5. (a, b) ANN's and LR's mean annual precipitation value. 
Table 2. Results.

\begin{tabular}{lccc}
\hline $\begin{array}{l}\text { Station } \\
\text { ID }\end{array}$ & $\begin{array}{c}\text { Actual } \\
\text { value }(\mathrm{mm})\end{array}$ & $\begin{array}{c}\text { LR predicted } \\
\text { value }\end{array}$ & $\begin{array}{c}\text { ANN predicted } \\
\text { value }\end{array}$ \\
\hline 1 & 815 & 859 & 827 \\
2 & 1382 & 1148 & 1407 \\
3 & 1266 & 1187 & 1286 \\
4 & 999 & 1245 & 1040 \\
5 & 891 & 1228 & 895 \\
\hline
\end{tabular}

Table 3. Performance - linear regression and ANN.

\begin{tabular}{lccc}
\hline Method & $\begin{array}{c}\text { Bias } \\
(\mathrm{mm})\end{array}$ & $\begin{array}{c}\text { RMSE } \\
(\mathrm{mm})\end{array}$ & Corr \\
\hline Linear regression & -62.8 & 217.72 & 0.3906 \\
ANN & -20.4 & 23.94 & 0.9986 \\
\hline
\end{tabular}

where, $P_{\text {val }}=$ precipitation value $(\mathrm{mm}), c_{\mathrm{o}}=$ precipitation value at sea level, $c_{1}=$ dimensionless rate of increase in rainfall with altitude, or height coefficient $(\mathrm{mm} / \mathrm{m}), E_{\text {st }}=$ station altitude $(\mathrm{m})$. Applying the formula to the data they found an overall relation:

$$
P_{\mathrm{val}}=672.49+\left((0.6439) \times E_{\mathrm{st}}\right) .
$$

The comparison has been performed between the actual precipitation values of the five observation stations that are within the Selinounda catchment area and the outcomes of the linear regression and ANN. The ANN gave the lowest MAPE value as can be seen in tables 2 and 3 .

\section{Conclusions}

In the present study, a multilayer feed-forward back-propagation neural network was developed to interpolate mean annual precipitation values of a specific catchment area in Achaia County, Greece. The ANN was capable of minimizing the effect of overfitting by monitoring in parallel the validation data during the training phase and terminating the process of learning when a certain criteria was achieved.

The performance and the estimate of error results for the interpolating precipitation data had an acceptable level of accuracy, while the comparison of a linear regression approach with the ones produced by this study showed that the ANN gave a better estimation. In particular, the outcomes of the study outlined the important advantage of the ANN against other interpolating methods since they neither require the specification of the function being modelled (e.g., linearity) nor make any underlying statistical assumptions about the data (e.g., normality). The generated continuous surface showed in more detail the spatial distribution of precipitation in the specific catchment area of Selinounda, resulting in a better estimate than linear regression analysis did. It can be concluded that the implementation of ANN techniques as a spatial interpolation method is a technique that improves the accuracy of analysis concerning hydrology and hydrogeological models, ground water studies, flood related applications and climate analysis studies, making them useful tools for local authorities and government agencies.

\section{References}

Amari S, Murata N, Muller K-R, Finke M and Yang H H 1997 Asymptotic statistical theory of overtraining and cross-validation; IEEE Trans. Neural Networks 8(5) 985-996.

Benardos A G and Kaliampakos D C 2004 A methodology for assessing geotechnical hazards for TBM tunnelingillustrated by Athens Metro, Greece; Int. J. Rock. Mech. Min. Sci. 41(4) 987-999.

Bryan B A and Adams J M 2002 Three-dimensional neurointerpolation of annual mean precipitation and temperature surfaces for China; Geogr. Anal. 34 93-111.

Chu S L, Zhou Z Y, Yuan L and Chen Q G 2008 Study on spatial precipitation interpolation methods; Pratacult Sci. 25(6) 19-23.

Daly C, Neilson R P and Phillips D L 1994 A statisticaltopographic model for mapping climatological precipitation over mountainous terrain; J. Appl. Meteorol. 33 $140-158$.

Garrett J H Jr 1994 Where and why artificial neural networks are applicable in civil engineering; J. Comput. Civil Eng. ASCE 8(2) 129-130.

Goncalves A M and Alpuim T 2006 Precipitation measurement and the analysis of hydrological resources in a river basin; In: 7th International Symposium on spatial accuracy assessment in natural resources and environmental sciences, pp. 851-860.

Goovaerts P 2000 Geostatistical approaches for incorporating elevation into the spatial interpolation of rainfall; J. Hydrol. 228 113-129.

Hagan T M, Demuth B H and Beale H M 1996 Neural network design; Electrical Engineering Series, Brooks/Cole, $730 \mathrm{p}$.

Hofstra N, Haylock M, Mew M, Jones P and Frei C 2008 Comparison of six methods for the interpolation of daily European climate data; J. Geophys. Res. 113 D21110, doi: 10.1029/2008JD010100.

Hutchinson P 1968 An analysis of the effect of topography on rainfall in the Taieri catchment area, Otago; Earth Sci. J. 2 51-68.

Jeffrey S J, Carter J O, Moodie K B and Beswick A R 2001 Using spatial interpolation to construct a comprehensive archive of Australian climate data; Environ. Model Softw. 16(4) 309-330.

Kajornrit J, Wong W K and Fung C C 2012 Estimation of missing precipitation records using modular Artificial Neural Networks (eds) Huang T et al., ICONIP 2012, Part IV, LNCS 7666, pp. 52-59.

Kohavi R 1995 A study of cross-validation and bootstrap for accuracy estimation and model selection; Proc. 14th Int. Joint Conf. on Artificial Intelligence (San Mateo: Morgan Kaufmann) 2(12) 1137-1143. 
Kong Y F and Tong W W 2008 Spatial exploration and interpolation of the surface precipitation data; Geogr. Res. 27(5) 1097-1108.

Kyriakidis P C, Kim J and Miller N L 2001 Geostatistical mapping of precipitation from rain gauge data using atmospheric and terrain characteristics; Am. Meteorol. Soc. 40 1855-1877.

Lloyd C D 2005 Assessing the effect of integrating elevation data into the estimation of monthly precipitation in Great Britain; J. Hydrol. 308 128-150.

Maren A, Harston C and Pap R 1990 Handbook of neural computing applications; Academic Press, San Diego, California.

Martínez-Cob A 1995 Estimation of mean annual precipitation as affected by elevation using multivariate geostatistics; Water Resour. Manag. 9 139-159.

Naoum S and Tsanis I K 2004 A multiple linear regression GIS module using spatial variables to model orographic rainfall; J. Hydroinform. 6 39-56.

Nourani V, Alami M T and Aminfar M H 2009 A combined neural-wavelet model for prediction of Lighvanchai watershed precipitation; Eng. Appl. Artif. Intell. 16 1-12.

Openshaw S and Openshaw C 1997 Artificial Intelligence in Geography (Chichester: John Wiley and Sons Ltd.)

Rigol J P, Jarvis C H and Stuart N 2001 Artificial neural networks as a tool for spatial interpolation; Int. J. Geogr. Inf. Sci. 15 323-343.

Rojas R 1996 Neural networks: A systematic introduction; Springer-Verlag, Berlin.

Rozos D, Koukis G, Sabatakakis N and Markantonis K 2008 Management of surface waters in the Achaia County with the constriction of water reservoir works; 8th International Hydrogeological Conference 2 705-722 (in Greek).

Rumelhart D E, Hinton G E and Williams R J 1986 Learning representations by back-propagating errors; Nature $\mathbf{3 2 3}$ $533-536$.

Shahin M A, Maier H R and Jaksa M B 2004 Data division for developing neural networks applied to geotechnical engineering; J. Comput. Civil Eng., ASCE 18(2) $105-114$.

Sivapragasam C, Liong S Y and Pasha M F K 2001 Rainfall and discharge forecasting with SSA-SVM approach; J. Hydroinform. 3(7) 141-152.

Snell S, Gopal S and Kaufmann R K 2000 Spatial interpolation of surface air temperature using artificial neural networks: Evaluating their use for downsizing GCMs; J. Climate 13 886-895.

Vicente-Serrano S M, Saz M A and Cuadrat J M 2003 Comparative analysis of interpolation methods in the middle Ebro valley (Spain): Application to annual precipitation and temperature; Clim. Res. 24 161-180.

Vuglinski V S 1972 Methods for the study of laws for the distribution of precipitation in medium-high mountains (illustrated by the Vitim River Basin), Distribution of precipitation in mountainous areas, WMO Publ. 326(2), World Meteorological Organization, Geneva, pp. $212-221$.

Wilk J, Kniveton D, Andersson L, Layberry R, Todd M C, Hughese D, Ringrose F and Vanderpost C 2006 Estimating rainfall and water balance over the Okavango River Basin for hydrological applications; J. Hydrol. 331(1-2) $18-29$. 